\title{
10000 Cerebral Aneurysm Coil Embolization Technical with a Coil-Assisted Technique Using a Small-Diameter Helical Coil
}

Naotsugu Toki, Hiroyuki Matsumoto, Hirokazu Nishiyama, and Daisuke Izawa

Objective: We introduce a coil-assisted technique using a small diameter helical coil to preserve a branch artery in the aneurysm neck or dome during coil embolization of a cerebral aneurysm.

Case Presentations: We report three cases that were treated with the coil-assisted technique. Using this method, the branch artery was preserved with a small diameter helical coil that was placed to support another frame coil. The first case was a ruptured internal carotid artery-posterior communicating artery (IC-Pcom) aneurysm, the second case was a ruptured anterior communicating artery aneurysm, and the third case was an unruptured IC-Pcom aneurysm, with branching of the Pcom, A2, and Pcom, respectively, from the neck or dome of the aneurysm. We were able to preserve the branch artery in all cases.

Conclusion: This technique is feasible and safe for coil embolization of intracranial branch-incorporated aneurysms. The technique is especially useful for preserving branch arteries that are difficult to preserve by conventional techniques.

Keywords > coil-assist technique, intracranial aneurysm, coil embolization

\section{Introduction}

In cerebral aneurysm coil embolization, several methods have been proposed to preserve a branch comes out from the neck or dome of aneurysm. The coil-assisted technique proposed by us is a branch-conserving method supporting the frame coil by placing a small-diameter helical coil as an assist coil near to the orifice of the branch. This technique may be useful in cases with difficulty in conserving the branch by conventional methods. We present cases treated with the coil-assisted technique at our hospital, and report its usefulness and safety with a literature review.

\section{Case Presentations}

This study was approved by the ethics committee of Kishiwada Tokushukai Hospital.

Department of Neurological Surgery, Kishiwada Tokushukai Hospital, Kishiwada, Osaka, Japan

Received: February 4, 2021; Accepted: August 10, 2021 Corresponding author: Naotsugu Toki. Department of Neurological Surgery, Kishiwada Tokushukai Hospital, 4-27-1, Kamoricho, Kishiwada, Osaka 596-8522, Japan Email: toki.naotsugu@gmail.com

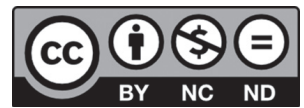

This work is licensed under a Creative Commons Attribution-NonCommercialNoDerivatives International License.

(C)2021 The Japanese Society for Neuroendovascular Therapy
We outline the coil-assisted technique performed by us. This procedure is an application of the double catheter technique. First, a microcatheter is guided into the dome in the aneurysm and another microcatheter is guided near to the branch. To guide the microcatheter exactly near to the branch, small shaping of the microcatheter tip is performed in many cases using the method that we previously reported. ${ }^{1)}$ An appropriate framing coil is inserted from the microcatheter in the dome and a small-diameter helical coil is placed near to the branch as an assist coil through the other microcatheter. At this time, it is important to intentionally place a small-diameter helical coil like a small cylinder by layering the coil loop in a concentric manner while avoiding covering the orifice and entangling the other frame coil. We normally use $2 \mathrm{~mm}-4 \mathrm{~cm}$ Target Helical US (Stryker, Kalamazoo, MI, USA) as an assist coil. As this is a small-diameter helical coil, the cylinder-like formation prepared by the assist coil may not be crushed by the frame coil. The frame coil is detached without detaching the assist coil, and subsequently, some coils are filled inside the frame coil as in the standard procedure. In general, the assist coil is detached after confirming that the frame and assist coils are stabilized by each other and the assist coil has no negative influence on the branch. When the assist coil becomes unnecessary or covers the branch during the procedure, retrieval of the assist coil is possible. When it is retrieved, the retrieved assist coil is used to filling the frame in the final step (Fig. 1). 
Toki $N$, et al.

Of 286 patients with ruptured and unruptured cerebral aneurysms treated by coil embolization at our hospital between January 2015 and June 2020, 3 were treated with the coil-assisted technique. The patients had a ruptured internal carotid artery-posterior communicating artery (IC-Pcom) aneurysm, ruptured anterior communicating artery (Acom) aneurysm, and unruptured IC-Pcom aneurysm, respectively. The Pcom, A2, and Pcom came out from the dome or near the neck, respectively, for which

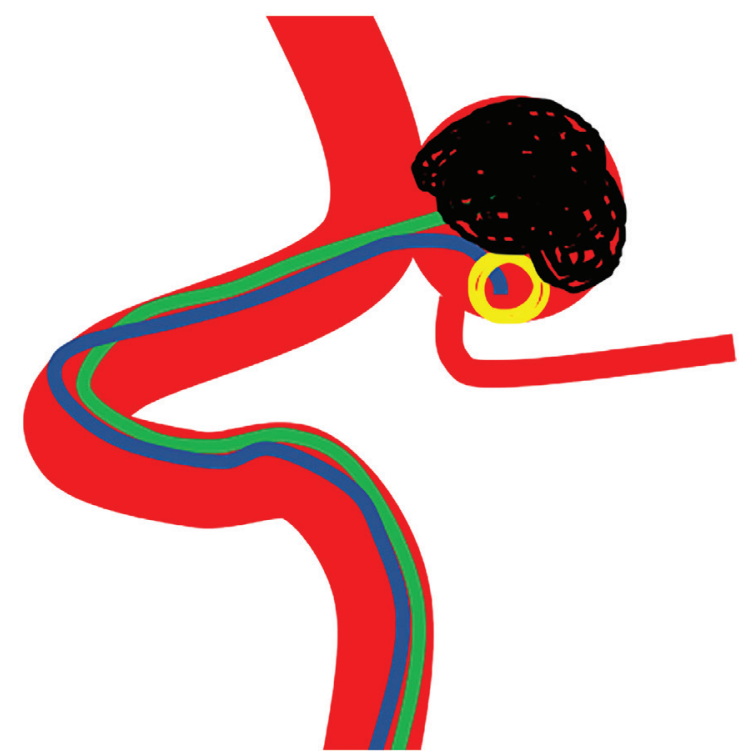

Fig. 1 Schema of the coil-assisted technique. Yellow circle is the assisted coil inserted from the microcatheter which is indicated with blue line. Black paint is the frame coil inserted from the microcatheter which is indicated with green line.
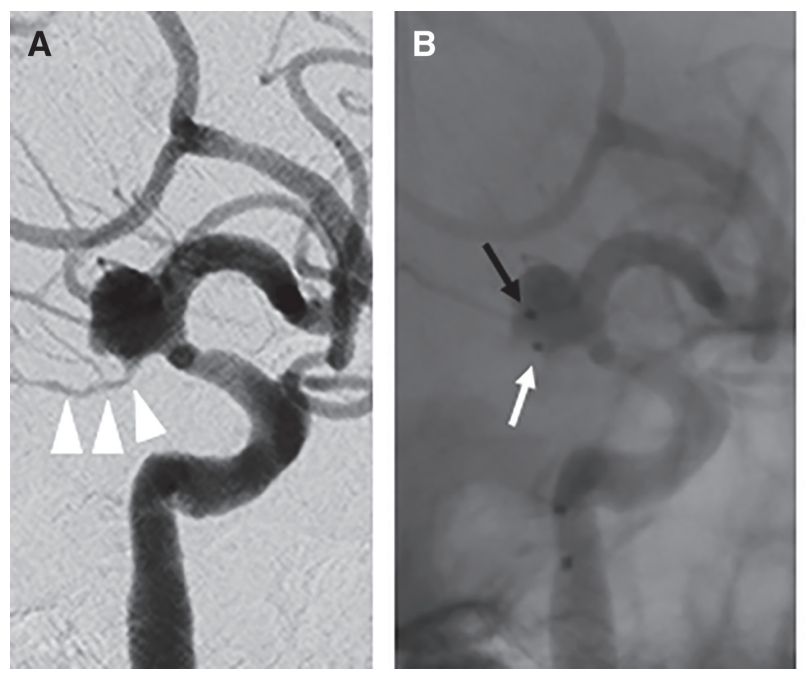

Fig. 2 (A) Preoperative fluoroscopic working view. The white arrowheads point to the Pcom that branched from the aneurysm dome. (B) The white arrow indicates the tip of the Echelon 10 catheter, which is ultra-small shaped and placed near the orifice of the branch artery, whereas the black arrow shows the tip of the Excelsior SL-10 preshaped $45^{\circ}$ catheter, which was placed in the body of the aneurysm. conservation of the branch by the conventional method was considered difficult; therefore, treatment was performed using the coil-assisted technique. The branch was able to be conserved in all 3 patients; there were no intraoperative or perioperative complications, and no recurrence has been observed during follow-up.

\section{Representative case 1}

Case 1 was an 82-year-old woman with a ruptured right IC-PC aneurysm (Fig. 2). The aneurysm was $6.5 \times 4.8 \times$ $6.1 \mathrm{~mm}$ in size and the neck diameter was approximately $5 \mathrm{~mm}$, being wide neck. The Pcom branched from the lower part of the aneurysmal dome, suggesting the necessity of an adjunctive technique to conserve the branch. No stent was used because this was a ruptured case and conservation of the branch by stenting or balloon herniation was considered difficult because the orifice was located in the dome part; thus, the coil-assisted technique was selected. Under general anesthesia, a 6-Fr Envoy (Johnson \& Johnson, New Brunswick, NJ, USA) was guided to the cervical region of the right internal carotid artery. First, an Excelsior SL-10 $45^{\circ}$ pre-shaped (Stryker) was guided to the body of the aneurysm, followed by guiding an Echelon 10 STR (2 mm of the tip was shaped into a moderate curve using a heat gun; Covidien, Dublin, Ireland) in order to place the tip near to the orifice of the branch. A Galaxy $4 \mathrm{~mm}-10 \mathrm{~cm}$ (Johnson \& Johnson) coil was inserted through the Excelsior SL-10 to prepare a frame, but it covered the orifice of the branch, and a Target Helical Ultrasoft
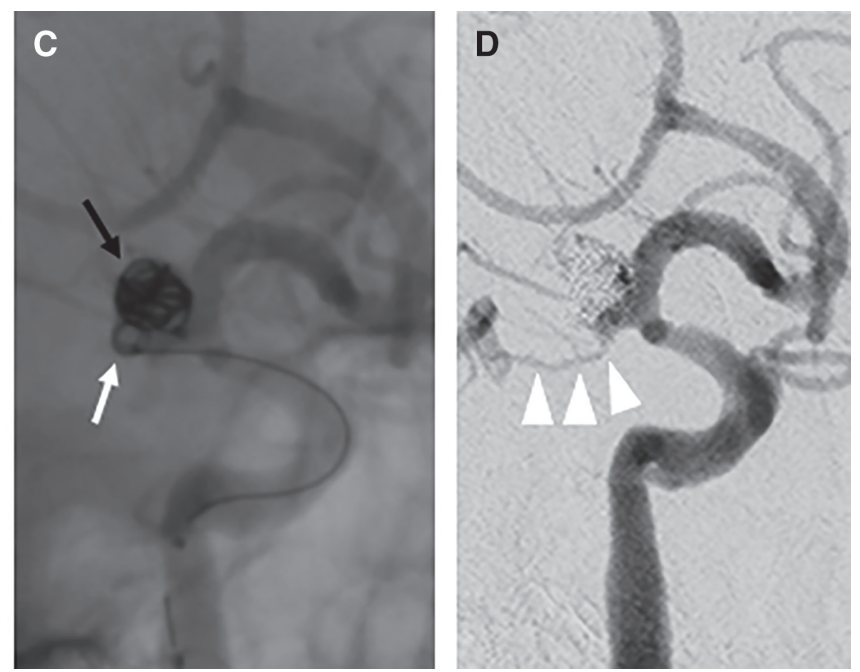

(C) A Target Helical Ultrasoft $2 \mathrm{~mm} \times 4 \mathrm{~cm}$ coil was inserted from the Echelon catheter as an assist coil (white arrow) and a Galaxy G3 4 $\mathrm{mm} \times 10 \mathrm{~cm}$ coil was inserted from the SL-10 catheter into the aneurysm as another frame coil (black arrow). (D) Postoperative angiography demonstrated aneurysm occlusion with preservation of the Pcom (arrowheads). Pcom: posterior communicating artery 

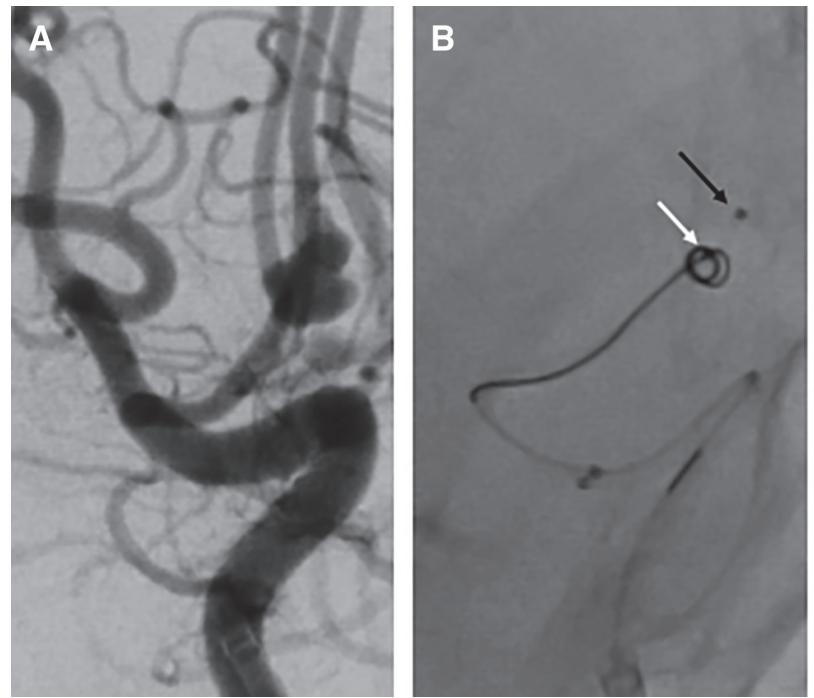

Fig. 3 (A) Preoperative down-the-barrel fluoroscopic view showing that the Acom aneurysm had an irregular shape. (B) The white arrow indicates the tip of the SL-10 microcatheter, which was placed in the bleb near the neck, and the Target Helical Ultrasoft $2 \mathrm{~mm} \times 4 \mathrm{~cm}$ coil, which was inserted into the bleb as an assist coil. The black arrow points to the tip of another SL-10 microcatheter, which was placed in

$2 \mathrm{~mm}-4 \mathrm{~cm}$ (Stryker) was subsequently inserted through the Echelon so as to form a small cylinder near to the orifice of the branch to prevent the frame from covering it. Then, inside the frame was filled with some coils and the assist coil was detached after confirming the stability of the coil mass. Finally, inside of assisted coil was also filled with tiny coils while conserving the Pcom and embolization was completed with a mild neck remnant. Complete occlusion was confirmed on cerebral angiography performed 1 year after treatment.

\section{Representative case 2}

Case 2 was a 61-year-old woman with a ruptured Acom aneurysm (Fig. 3). The aneurysm was $6.8 \times 5.9 \times 3.7 \mathrm{~mm}$ in size. It was lobular and divided into 3 lobes, and the neck encircled half of the Acom, being slightly wide neck. An adjunctive technique was considered necessary to preserve the bilateral A2 and perforating branch during embolization. No stent was used because it was a ruptured aneurysm, and the use of a balloon was considered a high risk because the parent blood vessel was thin. Thus, treatment with the coil-assisted technique was planned. Under general anesthesia, a 6-Fr Axcelguide (Medikit, Tokyo, Japan) was guided to the cervical region of the left internal carotid artery and a Cerulean DD6 (Medikit) was guided to the petrous part of the internal carotid artery as a middle catheter. Two Excelsior SL-10 STR were prepared. An approximately 2-mm small curve was prepared in the tip of
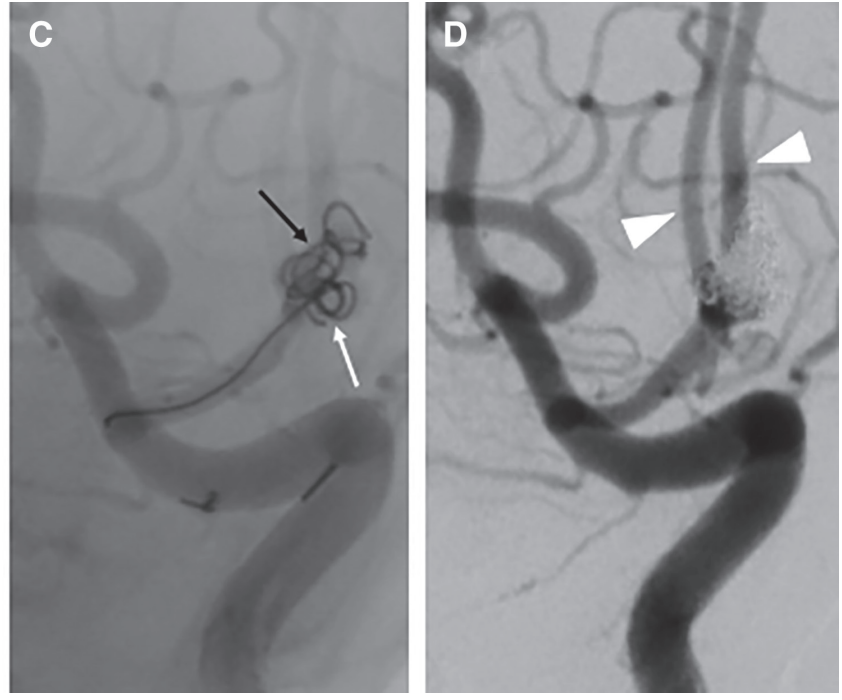

the body of the aneurysm. (C) The Target 360 Ultrasoft $3 \mathrm{~mm} \times 6 \mathrm{~cm}$ coil was inserted into another part of the aneurysm (black arrow) and was supported by the assist coil (white arrow). (D) Postoperative angiography demonstrated aneurysm occlusion with preservation of the Acom and A2 (white arrowheads). Acom: anterior communicating artery

one and guided into the bleb near the neck. The other Excelsior SL-10 was guided to the body of the aneurysmal dome. A Target Helical Ultrasoft $2 \mathrm{~mm}-4 \mathrm{~cm}$ was inserted to form a small cylinder through the Excelsior SL-10 guided into the bleb near the neck; a frame was prepared with a Target $360^{\circ}$ Ultrasoft $3 \mathrm{~mm}-6 \mathrm{~cm}$ (Stryker) coil through the other Excelsior SL-10. Inside the frame was filled with some coils, and the assist coil was detached because the frame became stable. Subsequently, inside the assist coil was also filled with tiny coils to conserve the Acom and A2, and embolization was completed. On follow-up cerebral angiography performed after 1 year, complete occlusion was confirmed.

\section{Discussion}

In this study, the coil-assisted technique was reported. This technique enables conservation of the branch by supporting the frame coil with a small-diameter helical coil that is placed like a small cylinder formation near to the orifice of the branch vessel.

Coil embolization for the treatment of cerebral aneurysms has recently been performed frequently compared with clipping. When the vessel branch comes out from the neck or the dome of an aneurysm, an adjunctive technique is necessary to conserve the branch in many cases, and several methods, such as balloon assist, stent assist, and catheter assist, ${ }^{2,3)}$ have been proposed. However, there are 
Toki $N$, et al.

often cases in which conservation of the branch by conventional methods is difficult. The coil-assisted technique is one of the adjunctive techniques, and several similar methods have been reported. Cho et al. and Ihn et al. reported a technique to conserve the branch coming out from the vicinity of the neck. ${ }^{4,5}$ According to the report, one of two catheters was guided to the origin of the branch, the coil was placed into the branch and left undetached, and the coil in the branch was then retrieved when the coil frame prepared with the other catheter was stabilized. However, this method may increase the risk of thrombus formation in the branch. Morioka et al. reported a scaffolding technique in which a microcatheter was guided to near the orifice of the branch and a coil was roughly wound around the orifice, which was later retrieved after a stable frame was formed with the other microcatheter in the aneurysm. ${ }^{\text {) }}$ However, this method may have risks such as thrombus formation while covering the branch with the coil, and entangling and unraveling the coil.

In the coil-assisted technique proposed by us, a microcatheter is not placed into the branch and the orifice is not covered with a coil. Instead, the microcatheter tip is guided to aside of the orifice of the branch and the other microcatheter is guided to the body of the aneurysmal dome. To accurately guide a microcatheter to the side of the branch, we use the ultra-small microcatheter tip shaping method that we previously reported. ${ }^{1)}$ A smalldiameter helical coil is inserted through the catheter guided to near the branch and wound into like a small cylinder to prepare a scaffold of the frame coil as an assist coil, and the coil is left undetached. For the smalldiameter coil, we use $2 \mathrm{~mm}-4 \mathrm{~cm}$ Target Helical Ultrasoft. Its hardness is considered appropriate, and it can be easily wound into like a small cylinder. In addition, as the diameter is small, the risks of deformation of the cylindrical form due to being crushed by the frame coil and entangling the frame coil may be unlikely. Through the other microcatheter, an appropriate frame and filling coils capable of homogenously filling the aneurysm should be placed. The assist coil placed aside the branch is detached when the assist coil and frame coil masses support each other and stabilize, and once conservation of the branch is confirmed, inside of the assist coil is also filled with small coils. When the assist coil becomes unnecessary during treatment, it can be retrieved without detachment. By winding a small-diameter helical coil like a small cylinder, the assist coil can be retrieved without interfering with or breaking the frame coil mass. When it is retrieved, it can be used as a coil for filling inside the other coil mass because it has a small diameter, thus not being wasted. We have not experienced a case of retrieval of the assist coil during treatment.

Unlike in previous reports, the branch is not covered and no foreign substance is placed into the branch, suggesting that the risks of thrombus formation and embolism are relatively low.

The coil-assisted technique using a small-diameter helical coil reported by us in this study may be relatively safe and useful for cases in which conservation of the branch is difficult, but more cases are necessary to demonstrate its efficacy and safety.

\section{Conclusion}

As an adjunctive technique, the coil-assisted technique using a small-diameter helical coil to conserve the branch in cerebral aneurysm coil embolization may be relatively safe and useful.

\section{Disclosure Statement}

The authors declare no conflicts of interest.

\section{References}

1) Matsumoto $H$, Nishiyama $H$, Tetsuo $Y$, et al. Ultra-small catheter shaping method with a sheath dilator: usefulness for coil embolization of cerebral aneurysms. JNET J Neuroendovasc Ther 2019; 13: 21-27.

2) Lai YJ, Yen $\mathrm{CH}$, Hsieh $\mathrm{LC}$, et al. A versatile method for treating intracranial wide-neck aneurysms: catheterassisted technique of three variations. Interv Neuroradiol 2010; 16: 394-399.

3) Luo CB, Chang FC, Lin CJ, et al. A coil placement technique to treat intracranial aneurysm with incorporated artery. J Chin Med Assoc 2018; 81: 255-261.

4) Cho YD, Lee JY, Seo JH, et al. Coil protection using small helical coils for wide-neck intracranial aneurysms: a novel approach. AJNR Am J Neuroradiol 2013; 34: 164-168.

5) Ihn YK, Kim BM, Suh SH, et al. Coil-protected embolization technique for a branch-incorporated aneurysm. Korean J Radiol 2013; 14: 329-336.

6) Morioka J, Murao K, Miyake K, et al. Scaffolding technique: a new double-catheter technique for coil embolization of wide-necked aneurysms. JNET J Neuroendovasc Ther 2019; 13: 183-188. 